Ann. Zootech., I979, 28 (3), 297-3I4.

\title{
Protéines alimentaires et qualité de l'œuf. \\ II. - Influence de la nature des protéines distribuées aux poules sur les caractéristiques sensorielles de l'œuf et la teneur en acides aminés libres du jaune
}

B. COLAS, F. SAUVAGEOT, J.-P. HARSCOAT ( ${ }^{1}$ ) et B. SAUVEUR (')

\author{
(') Station de Recherches Avicoles, \\ Centre de Recherches de Tours, I.N.R.A., \\ Nouzilly, 37380 Monnaie (France) \\ Laboratoire de Biologie Physico-Chimique \\ École Nationale Supérieure de Biologie Appliquée à la Nutrition \\ et à l'Alimentation (ENS.BANA), 2Iroo Dijon (France)
}

\begin{abstract}
Résumé
Les expériences ont été conduites dans le but d'étudier l'influence de la substitution partielle du tourteau de soja par de nouvelles protéines alimentaires dans la ration de poules pondeuses Warren sur les caractéristiques sensorielles des oufs et sur la teneur en acides aminés libres des jaunes.

Six régimes expérimentaux ont été répartis en 3 séries :

- pois (3o p. Iоo) et féverole (I5 p. IOO);

- farine de poisson (Io p. Ioo) et protéine de luzerne (6 p. Ioo);

- farine de viande (Iо p. гоo) et algues spirulines (II,5 p. IоO).

Chaque série inclue un régime témoin à base de tourteau de soja. Un jury entraîné évaluait les blancs et les jaunes d'œufs crus et à la coque en utilisant un questionnaire de type profil.

Les résultats obtenus montrent que :

I. L'incorporation dans la ration des animaux de farine de poisson, de protéine de luzerne ou d'algues spirulines, entraîne la production d'œufs présentant des caractéristiques sensorielles différentes et de qualité inférieure à celle des œufs produits par les animaux recevant le régime témoin. Inversement, l'incorporation de pois améliore les qualités sensorielles des œufs.

2. La forme de présentation des œufs modifie leurs caractéristiques sensorielles.

3. Au niveau des acides aminés libres, les variations sont faibles, tant aux niveaux qualitatif que quantitatif, et peuvent difficilement expliquer l'apparition de flaveurs anormales dans les jaunes.
\end{abstract}




\section{Introduction}

Depuis une dizaine d'années de nombreux travaux ont été consacrés à la recherche de nouvelles sources de protéines pouvant se substituer partiellement ou en totalité au tourteau de soja dans l'alimentation des animaux monogastriques. Parmi ces derniers, les poules pondeuses ont été largement utilisées et on connait assez bien aujourd'hui les effets de nombreuses sources de protéines sur le nombre d'œufs produits ou le poids moyen de l'ouf. L'action éventuelle de ces protéines sur les propriétés fonctionnelles (pouvoir moussant du blanc, pouvoir émulsifiant du jaune) et les caractéristiques sensorielles des œufs a été, semble-t-il, beaucoup moins étudiée.

Parmi les sources protéiques pouvant se substituer au tourteau de soja, deux semblent particulièrement à l'origine de flaveurs désagréables dans les œufs : les farines de poisson (KGHLER et BEARSE, I975) et les tourteaux de colza (Hawrysh et al., I975; OVERFIEld et Elson, I975; LEESON et SUmmers, I978). Les flaveurs de poisson ou de crabe provenant de l'incorporation de ces aliments sont attribuées à la présence de triméthylamine dans les oufs due à un excès de choline dans les farines de poisson et de sinapine dans les tourteaux de colza, et il est maintenant bien établi qu'elles sont fonction non seulement du taux d'incorporation de l'aliment protéique mais aussi de l'origine de celui-ci et de l'âge et de la race des poules pondeuses. Ainsi, l'incorporation dans la ration de farine de hareng altère plus la flaveur des œufs que celle de farine de colin, pour un même taux de 5 p. IOO (KOEHLER et BEARSE, I975). De plus, les modifications sont moins importantes au début du cycle de ponte que 5 mois plus tard, bien qu'il soit difficile de préciser, de 1'avis des auteurs eux-mêmes, si cette évolution est due au vieillissement des animaux ou à celui de la farine de poisson. Par ailleurs, Hawrysh et al. (I975) observent que des poules Rhode Islande Red recevant un régime contenant $6,8 \mathrm{p}$. Ioo de tcurteau de colza pondent des oufs ayant une flaveur désagréable alors que des poules Plymouth Rock blanches ou WHITE Leghorn recevant le même régime pondent des œufs ayant une flàveur normale.

La répercussion des traitements appliqués sur la qualité interne de l'œuf et sur ses propriétés fonctionnelles est rapportée par ailleurs (SAUvEUR, ZyBKo et Coras, r979).

La présente étude a été réalisée pour examiner si différentes sources protéiques peuvent être substituées, partiellement au moins, au tourteau de soja, sans modifier les caractéristiques sensorielles des œufs. Les matières protéiques retenues ont été les suivantes : 3 protéines végétales (légumineuses pois et féverole et concentré de jus de luzerne), 2 protéines animales (farines de viande et de poisson), une protéine d'organisme unicellulaire (algues Spirulines).

La répercussion de ces régimes sur la teneur en acides aminés libres du jaune a également été examinée. La teneur du jaune en acides aminés dépend, en effet, de la composition azotée de l'aliment consommé par la poule pondeuse; les variations sont cependant faibles. La contribution de ces variations aux modifications de la flaveur des jaunes d'œufs ne semble pas avoir été étudiée. Plus généralement, les relations entre acides aminés et flaveur des aliments restent mal connues, d'autant que la contribution d'un mélange de plusieurs acides aminés à la flaveur d'un aliment dépasse souvent la somme de celles de chacun des acides aminés à l'état pur en raison de l'existence d'effets synergiques ou potentiateurs. Solms (I969) précise cependant la flaveur des acides aminés en solution pure et les 
classe en 3 groupes, ceux n'ayant aucun goût, ceux ayant une saveur amère ou sucrée, et cełıx ayant une flaveur complexe. Ce troisième groupe comprend l'acide glutamique et les acides aminés soufrés (cystéine et méthionine). Plus récemment, HIPpe et WARTHESEN (I978) associent l'odeur sulfureuse de quelques aliments supplémentés en méthionine à la dégradation de celle-ci par traitement thermique.

\section{Matériel et méthodes}

Six régimes expérimentaux ont été répartis en 3 séries de 2 régimes, chaque série comprenant également un régime témoin (I $4,3 \mathrm{p}$. Ioo de tourteau de soja). La répartition en 3 séries a été effectuée selon le schéma suivant :

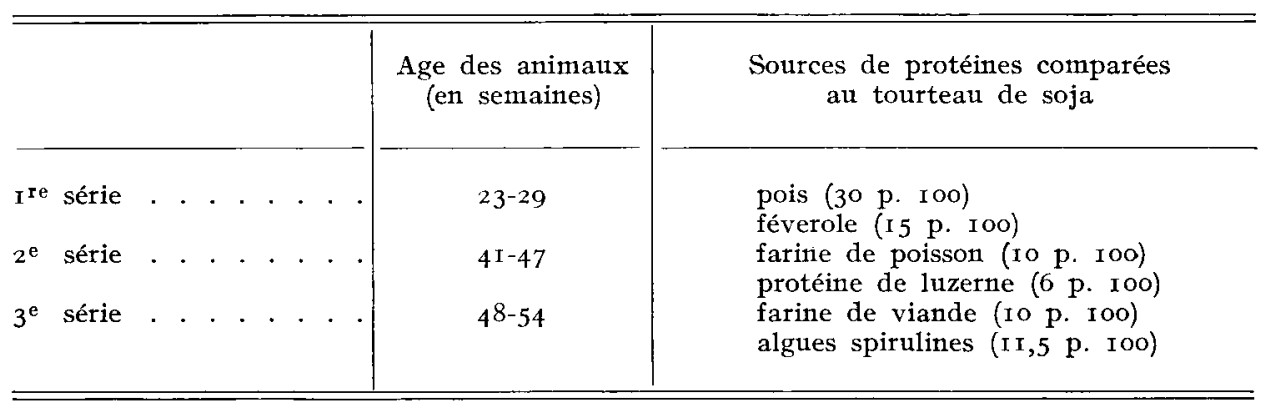

Chaque régime expérimental a été distribué à 48 poules WARREN IsA élevées à la station de recherches avicoles (I.N.R.A., Nouzilly) .

Lorsque le niveau d'incorporation de chaque source protéique ne permettait pas d'atteindre un contenu en matières azotées totales du régime égal à I5o $\mathrm{g} / \mathrm{kg}$, le complément a été assuré dans tous les cas par du tourteau de soja. Tous les régimes ont été calculés pour apporter les mêmes quantités d'énergie métabolisable, protéines, lysine, méthionine, calcium et phosphore. Leur composition détaillée et les conditions d'environnement des animaux sont données par SaUveur, Zybko et Colas (I979). Entre les séries I et 2, les animaux ont reçu un aliment commercial de type "poule pondeuse ».

\section{Évaluation sensorielle des auts}

Les caractéristiques sensorielles des œufs (blanc et jaune) ont été évaluées par un jury de 5 membres, choisis au hasard parmi 8 éléments $(7$ hommes et I femme) préalablement caractérisés du point de vue sensoriel et entraînés à l'évaluation des oufs, en particulier à la détection de l'odeur et de la flaveur de poisson.

La qualité des œufs pouvant varier en fonction des conditions de conservation (température et durée en particulier), les œufs ont été conservés, avant évaluation, $48 \mathrm{~h}$ à température ambiante puis 24 ou $48 \mathrm{~h}$ (selon les contraintes expérimentales) à $+4^{\circ} \mathrm{C}$.

Les œufs ont été évalués sous 2 formes : cru et à la coque. Pour les œufs 
crus, les juges cassaient eux-mêmes l'échantillon sur une assiette et évaluaient le blanc puis le jaune. Pour les oufs à la coque, les échantillons étaient présentés aux juges immédiatement après cuisson. La cuisson à la coque était obtenue en $3,50 \mathrm{mn}$ dans l'eau bouillante, les œufs étant maintenus immergés verticalement, pointe en bas, dans un panier métallique. Les juges évaluaient le blanc puis le jaune.

\section{ÉVALUATION SENSORIELIE DU BLANC D'CEUF CRU}

Test No

Dat

Nom

ODEUR

: L'odeur d'œuf est-elle franche?

OUI $\square$ NON

Si oUI, quelle est son intensité?

..........

Si NoN, quelle est la thature de l'odeur (ou des odeurs) parasites :

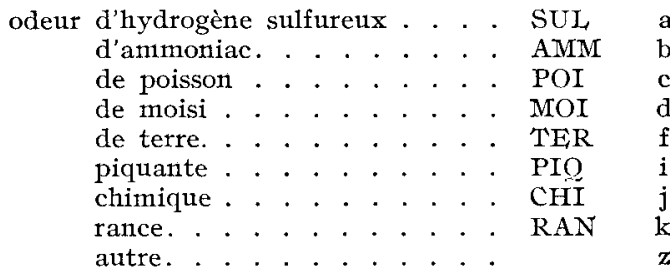

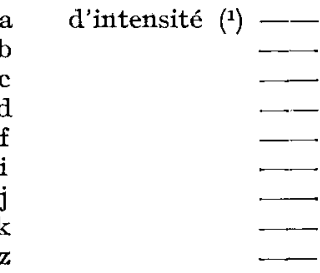

FI,AVE,UR

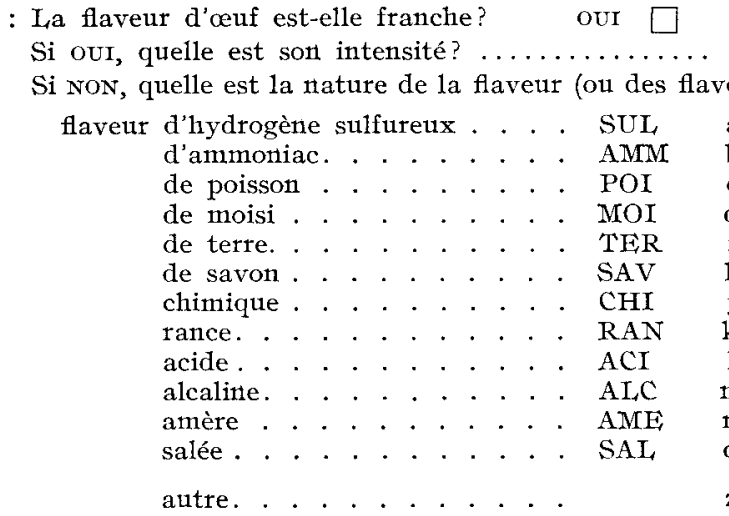

NON

s) parasites?

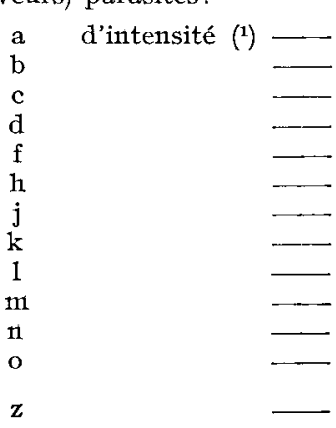

ARRIÈrRE-GOUT : Existe-t-il un ou plusieurs arrière-goût(s) désagréable(s)?

Si our, indiquez-en la nature $\ldots \ldots \ldots$ et l'intensité ${ }^{(1)}$

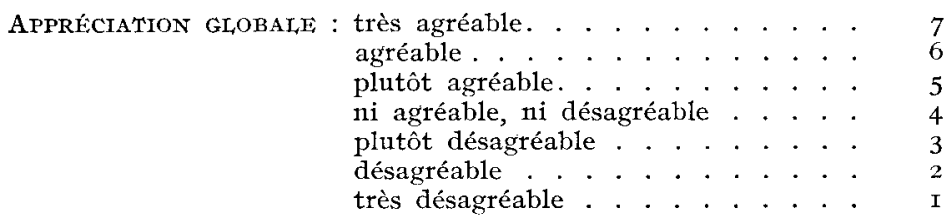

(1) Intensité du défaut : $4=$ fort, $3=$ plutôt fort, $2=$ ni faible, ni fort, $I=$ faible.

FIG. I. - Questionnaire utilisé pour l'évaluation sensorielle des aufs (type blanc cru). Record sheet used for taste panel evaluation of eggs, raw white kind. 
Pour chaque série, les œufs ont été examinés après 4 et 6 semaines d'adaptation des animaux aux régimes expérimentaux, avec répétition sur 2 jours consécutifs. Au cours d'une séance, et pour les 2 formes de présentation (œuf cru et œuf à la coque), chaque juge évaluait les 3 échantillons de la série ( $\mathrm{r}$ témoin et 2 régimes expérimentaux) codés et présentés au hasard. L'ordre d'évaluation des 2 formes de présentation variait entre les 5 juges au cours d'une même séance et, pour un juge, entre les 2 répétitions.

Les évaluations ont été effectuées dans une salle spécialement équipée. A cet effet, chaque stalle était éclairée en lumière rouge afin de masquer les différences de couleur entre les jaunes des différents échantillons. Les juges disposaient de pain et d'eau minérale (Volvic) pour supprimer les flaveurs résiduelles de la bouche entre les échantillons d'œufs.

Un questionnaire de type profil, établi d'après celui décrit par KOEHLER et Jacobson (I972), a été utilisé. Dans cette méthode d'analyse descriptive, le blanc et le jaune d'un échantillon sont évalués successivement du point de vue odeur, flaveur et arrière-goût (fig. I).

Dans une même série, pour un juge et pour une forme de présentation donnés, un classement entre les 3 échantillons a été réalisé sur la base du dénombrement des caractéristiques sensorielles franches (odeur, flaveur et arrière-goût), le rang I (échantillon le mieux classé) étant attribué à l'échantillon présentant le nombre de caractéristiques sensorielles franches le plus élevé. En cas d'égalité, les échantillons ont été départagés au moyen des notes d'appréciation globale, le rang I étant attribué à 1'échantillon ayant la note la plus élevée. Pour la première série expérimentale, la validité de ce mode de classement a été éprouvée par comparaison avec un questionnaire de classement dans lequel il était demandé au juge de classer les 3 échantillons en allant du plus franc au moins franc. Pour les deuxième et troisième séries, seule la technique du profil a été utilisée.

\section{Dosage des acides aminés libres du jaune}

Les teneurs en acides aminés libres des jaunes d'œufs ont été déterminées le jour même de la ponte selon la technique décrite par LARBIER (I973). Après extraction à 1'acide picrique à I p. Ioo, la séparation et le dosage des acides aminés libres ont été effectués par chromatographie sur colonne de résine échangeuse d'ions selon la méthode de Moore, Spackman et Stein (I958). Pour chaque régime, 3 dosages ont été effectués, chacun sur un mélange de ro jaunes.

\section{Résultats}

Dans tout ce qui suit, on appellera régime "pois", "féverole", etc... les régimes dont la source de protéine autre que le tourteau de soja est constituée respectivement par la farine de pois, de féverole, etc...

\section{Caractéristiques sensorielles des oufs}

Les résultats de chacune des 3 séries sont donnés dans les tableaux I à 6 .

Les tableaux $\mathrm{r}, 3$ et 5 donnent les sommes des rangs et le classement des 3 types d'œufs pour chacune des 3 séries. La somme des rangs la plus faible corres- 
TABLEAU I

${ }^{\mathrm{re}}$ série: classement des aufs

Ist experiment: egg ranking

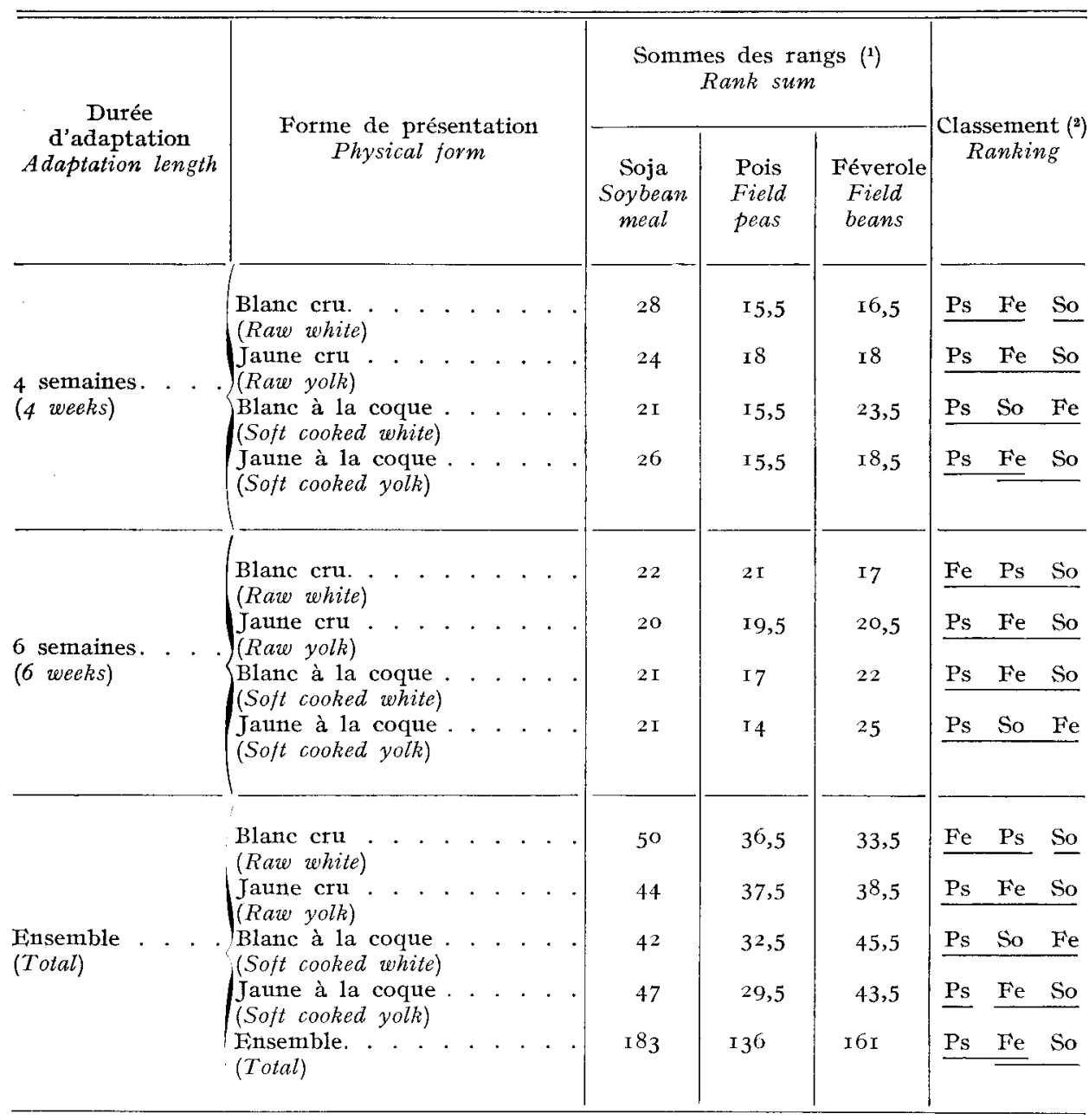

(1) Pour 4 et 6 semaines, chaque valcur est la somme de ro réponses. Ira somme des rangs la plus faible correspond à l'échantillon ayant les caractéristiques sensotielles les plus franches (For 4 and 6 teeeks, every data is the sum of to judgments. The lowest rank sum is for the sample with the cleanest sensory chavacteristics).

${ }^{(2)}$ L.es essais soulignés d'un même trait ne diffèrent pas significativement $(\mathbf{P} \leqslant 0,05)$ (Samples underscored by the same line do not differ significantly $(P \leqslant 0,05))$.

So $=$ Soja $($ Soybean meal $) ;$ Ps $=$ Pois (Field peas); Fe = Féverole (Field beans).

pond à l'échantillon ayant les caractéristiques sensorielles les plus franches. Ces résultats sont donnés pour les 2 durées d'adaptation des animaux aux régimes expérimentaux (4 et 6 semaines) et pour les 4 formes de présentation des œufs (blanc cru, blanc à la coque, jaune cru et jaune à la coque). Les sommes des rangs 
TABLEAU 2

$I^{\text {re }}$ série: caractéristiques sensorielles des au/s (')

rst experiment: sensory characteristics of eggs

\begin{tabular}{|c|c|c|c|c|c|}
\hline \multirow{2}{*}{$\begin{array}{c}\text { Forme } \\
\text { de présentation } \\
\text { Physical form }\end{array}$} & \multirow[t]{2}{*}{$\begin{array}{l}\text { Régime } \\
\text { Diet }\end{array}$} & \multirow{2}{*}{$\begin{array}{l}\text { Nombre } \\
\text { d'ouufs ne } \\
\text { présentant } \\
\text { aucun } \\
\text { défaut } \\
\text { Number } \\
\text { of eggs } \\
\text { without } \\
\text { taint }\end{array}$} & \multicolumn{2}{|c|}{$\begin{array}{l}\text { Nombre de défauts } \\
\text { et défauts principaux } \\
\text { Number of taints } \\
\text { and main taints }\end{array}$} & \multirow{2}{*}{$\begin{array}{l}\text { Appré- } \\
\text { ciation } \\
\text { globale } \\
(\mathbf{3}) \\
\text { Palatability } \\
\text { score }\end{array}$} \\
\hline & & & $\begin{array}{c}\text { Odeur }\left({ }^{2}\right) \\
\text { Odour }\end{array}$ & $\begin{array}{l}\text { Flaveur }\left({ }^{2}\right) \\
\text { Flavour }\end{array}$ & \\
\hline $\begin{array}{l}\text { Blanc cru. . . } \\
\text { (Raw white) }\end{array}$ & 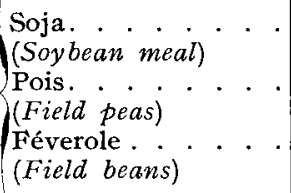 & $\begin{array}{l}2 \\
4 \\
6\end{array}$ & $\begin{array}{l}5 / 3 \text { poi } 3 \\
2 /- \\
I /-\end{array}$ & $\begin{array}{l}9 / 3 \text { poi } 3,3 \\
\text { et } 3 \text { chi } 2 \\
7 /- \\
4 /-\end{array}$ & $\begin{array}{l}3, \mathrm{I} \\
3,7 \\
3,8\end{array}$ \\
\hline $\begin{array}{l}\text { Jaune cru. . . . } \\
\text { (Raw yolk) }\end{array}$ & 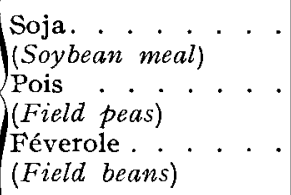 & $\begin{array}{l}4 \\
8 \\
6\end{array}$ & $\begin{array}{l}\mathrm{I} /- \\
- \\
\mathrm{I} /-\end{array}$ & $\begin{array}{l}8 /- \\
2 /- \\
6 /-\end{array}$ & $\begin{array}{l}3,9 \\
4,8 \\
4,7\end{array}$ \\
\hline $\begin{array}{l}\text { Blanc à la coque } \\
\text { (Soft cooked white) }\end{array}$ & $\begin{array}{l}\text { Soja. . · . . } \\
(\text { Soybcan meal }) \\
\text { Pois. . . . . } \\
(\text { Field peas }) \\
\text { Féverole. . . . . } \\
(\text { Field beans })\end{array}$ & $\begin{array}{l}4 \\
5 \\
\text { I }\end{array}$ & $\begin{array}{l}3 /- \\
3 /- \\
5 /-\end{array}$ & $\begin{array}{l}9 / 4 \text { ter } 2 \\
6 /- \\
8 /-\end{array}$ & $\begin{array}{l}4, \mathrm{I} \\
4,0 \\
3,8\end{array}$ \\
\hline $\begin{array}{l}\text { Jaune à la coque } \\
\text { (Soft cooked yolk) }\end{array}$ & 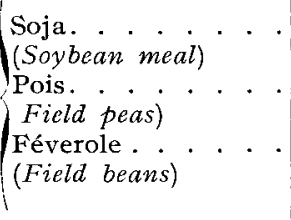 & $\begin{array}{l}5 \\
6 \\
5\end{array}$ & $\begin{array}{l}3 /- \\
1 /- \\
4 /-\end{array}$ & $\begin{array}{l}6 /- \\
4 /- \\
7 /-\end{array}$ & $\begin{array}{l}5,0 \\
5,0 \\
4,3\end{array}$ \\
\hline
\end{tabular}

(1) Valeurs obtenues à partir de ro réponses (Data calculated from Io judgments).

(2) Pour chaque essai sont mentionnés : le nombre total de défauts/les défauts principaux : nombre, nature et intensité moyenne (voir nature et échelle figure I) (For each sample: the total number of tainis ) the main taints: number, kind and average intensity (see kind and scale figure 1 ), are indicated).

(3) Voir échelle figure I (See scalo figure I).

ont été comparées au moyen de 1'épreuve de KRAMER et al. (I974). En présence de différences significatives $(P \leqslant 0,05)$, les contrastes entre échantillons pris 2 à 2 ont été examinés à 1'aide de la technique de la P.P.D.S. (plus petite différence significative). 


\section{TABLEAU 3}

$2^{\mathrm{e}}$ série: classement des aufs

and experiment: egg ranking

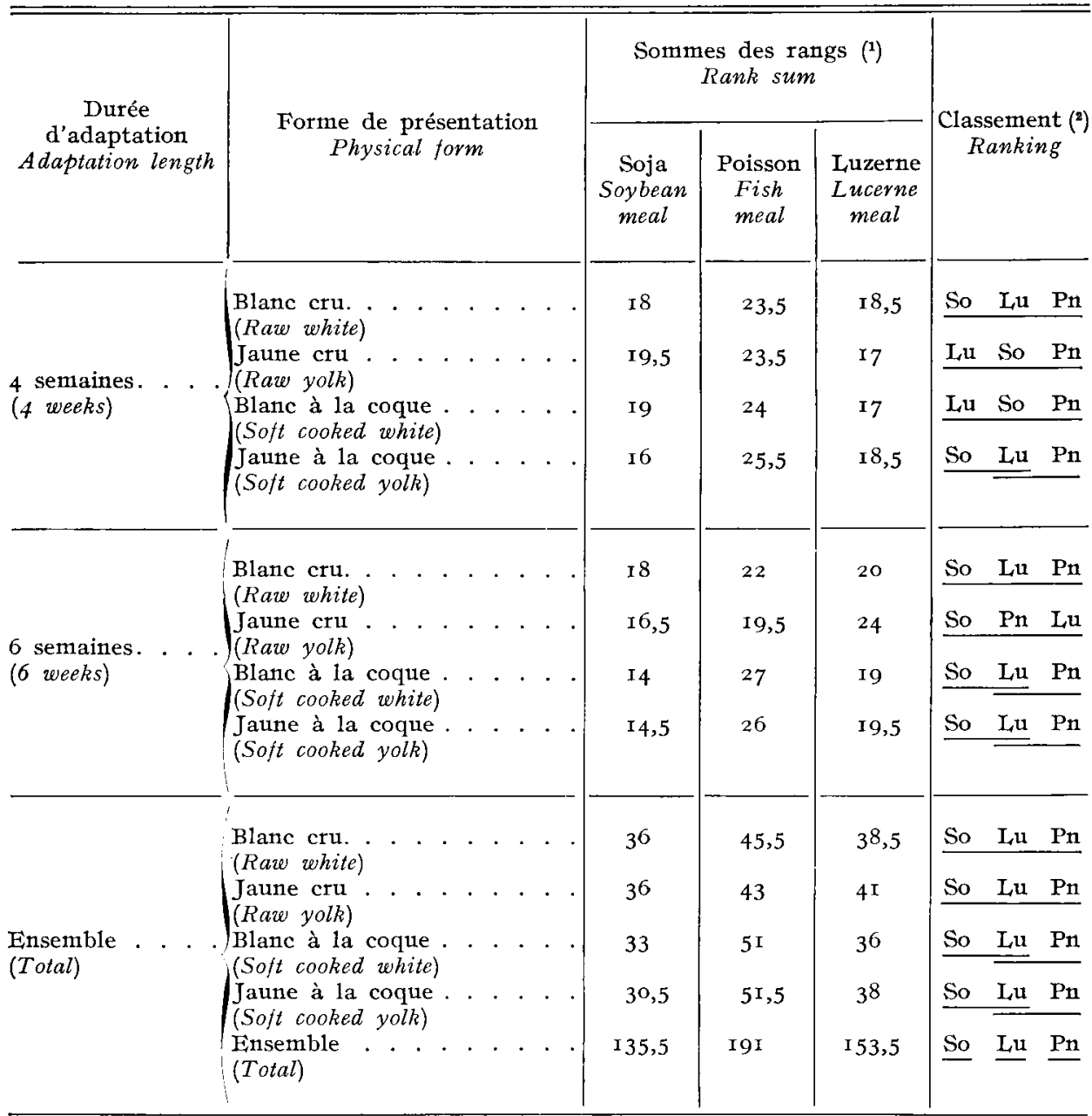

$\left({ }^{1}\right)$ et $\left({ }^{2}\right)$ Voir tableau I (See table $\left.\mathrm{I}\right)$; So $=$ Soja (Soybean meal); Pn = Poisson (Fish meal); L $\mathrm{u}=$ Luzerne (Lucerne meal).

Les tableaux 2, 4 et 6 donnent, pour les 3 types d'œufs de chacune des 3 séries, les nombres d'œufs ne présentant aucun défaut (d'odeur, de flaveur et d'arrière-goût), les principaux défauts (nombre, nature et intensité) et les notes d'appréciation globale. 


\section{TABLEAU 4}

$2^{\mathrm{e}}$ série: caractéristiques sensorielles des outs (1)

and experiment: sensory characteristics of eggs

\begin{tabular}{|c|c|c|c|c|c|}
\hline \multirow{2}{*}{$\begin{array}{c}\text { Forme } \\
\text { de présentation } \\
\text { Physical form }\end{array}$} & \multirow{2}{*}{$\begin{array}{l}\text { Régime } \\
\text { Diet }\end{array}$} & \multirow{2}{*}{$\begin{array}{c}\text { Nombre } \\
\text { d'œufs ne } \\
\text { présentant } \\
\text { aucun } \\
\text { défaut } \\
\text { Number } \\
\text { of eggs } \\
\text { without taint }\end{array}$} & \multicolumn{2}{|c|}{$\begin{array}{l}\text { Nombre de défauts } \\
\text { et défauts principaux } \\
\text { Number of taints } \\
\text { and main taints }\end{array}$} & \multirow{2}{*}{$\begin{array}{l}\text { Appré- } \\
\text { ciation } \\
\text { globale () } \\
\text { Palatability } \\
\text { score }\end{array}$} \\
\hline & & & $\begin{array}{l}\text { Odeur }(\mathbf{2}) \\
\text { Odour }\end{array}$ & $\begin{array}{l}\text { Flaveur }\left(^{2}\right) \\
\text { Flavour }\end{array}$ & \\
\hline $\begin{array}{l}\text { Blanc cru. } \\
\text { (Raw white) }\end{array}$ & $\left\{\begin{array}{l}\text { Soja. } \\
\text { (Soybean meal }) \\
\text { Poisson } \cdot \\
(\text { Fish meal }) \\
\text { Luzerne } \cdot \\
\text { (Lucerne meal) }\end{array}\right.$ & $\begin{array}{l}8 \\
5 \\
8\end{array}$ & $\begin{array}{l}5 / 3 \text { poi } 2,7 \\
8 / 5 \text { poi } 2,2 \\
3 /-\end{array}$ & $\begin{array}{l}\text { I2/5 poi } 2,6 \\
\text { I9/4 I poi } 2,2 \\
\text { I } 4 / 4 \text { poi } 3,3\end{array}$ & $\begin{array}{l}3,8 \\
3,4 \\
3,7\end{array}$ \\
\hline $\begin{array}{l}\text { Jaune cru. } \\
(\text { Raw yolk })\end{array}$ & $\begin{array}{l}\text { Soja. } \\
\text { (Soybean meal) } \\
\text { Poisson } \cdot \\
(\text { Fish meal) } \\
\text { Luzerne } \cdot \\
\text { (Lucerne meal) }\end{array}$ & $\begin{array}{l}\text { I6 } \\
\text { I } 5 \\
\text { I I }\end{array}$ & $\begin{array}{l}- \\
3 /- \\
2 /-\end{array}$ & $\begin{array}{l}6 /- \\
4 /- \\
\text { I I } / 3 \text { sal I,7 }\end{array}$ & $\begin{array}{l}5, \mathrm{I} \\
4,7 \\
4,9\end{array}$ \\
\hline $\begin{array}{l}\text { Blanc à la coque } \\
\text { (Soft cooked white) }\end{array}$ & $\begin{array}{l}\text { Soja. } \\
(\text { Soybean meal }) \\
\text { Poisson } \cdot \\
\left(\begin{array}{l}\text { (Fish meal }) . \\
\text { (Luzerne } . \cdot \\
\text { (Lucerne meal) }\end{array}\right.\end{array}$ & $\begin{array}{r}7 \\
\mathrm{I} 2\end{array}$ & $\begin{array}{l}3 /- \\
8 / 3 \text { ter } 2,7 \\
5 / 3 \operatorname{sul} 2,3\end{array}$ & $\begin{array}{l}5 /- \\
\text { I } / 4 \text { poi } 2,3 \\
5 /-\end{array}$ & $\begin{array}{l}3,8 \\
4,5\end{array}$ \\
\hline $\begin{array}{l}\text { Jaune à la coque } \\
\text { (Soft cooked yolk) }\end{array}$ & $\left\{\begin{array}{l}\text { Soja. } \\
(\text { Soybean meal }) \\
\text { Poisson } \\
(\text { Fish meal }) \\
\text { Luzerne } \\
\text { (Luceme meal) }\end{array}\right.$ & $\begin{array}{l}17 \\
6 \\
15\end{array}$ & $\left|\begin{array}{l}2 /- \\
\text { I I } / 5 \text { poi } 2,4 \\
2 /-\end{array}\right|$ & $\begin{array}{l}2 /- \\
14 / 5 \text { poi } 2,8 \\
4 /-\end{array}$ & $\begin{array}{l}3,7 \\
5, \mathrm{o}\end{array}$ \\
\hline
\end{tabular}

(1) Valeurs obtenues à partir de 20 réponses (Data calculated from 20 judgments).

(2) et $\left({ }^{3}\right)$ Voir tableau 2 (See table 2 ).

\section{Effet de la durée d'adaptation des animaux aux régimes}

Cet effet semble négligeable. En effet, si des différences sont parfois observées entre 4 et 6 semaines (tabl. 7), elles ne conduisent à aucun résultat contradictoire. Il a donc été jugé logique de regrouper, pour une même forme de présentation, les résultats correspondant aux 2 durées d'adaptation (tab1. 2,4 et 6 ). Les sommes des rangs pour l'ensemble des formes de présentation ont également été calculées. 


\section{TABLEAU 5}

$3^{\mathrm{e}}$ série: classement des outs

3rd experiment: egg ranking

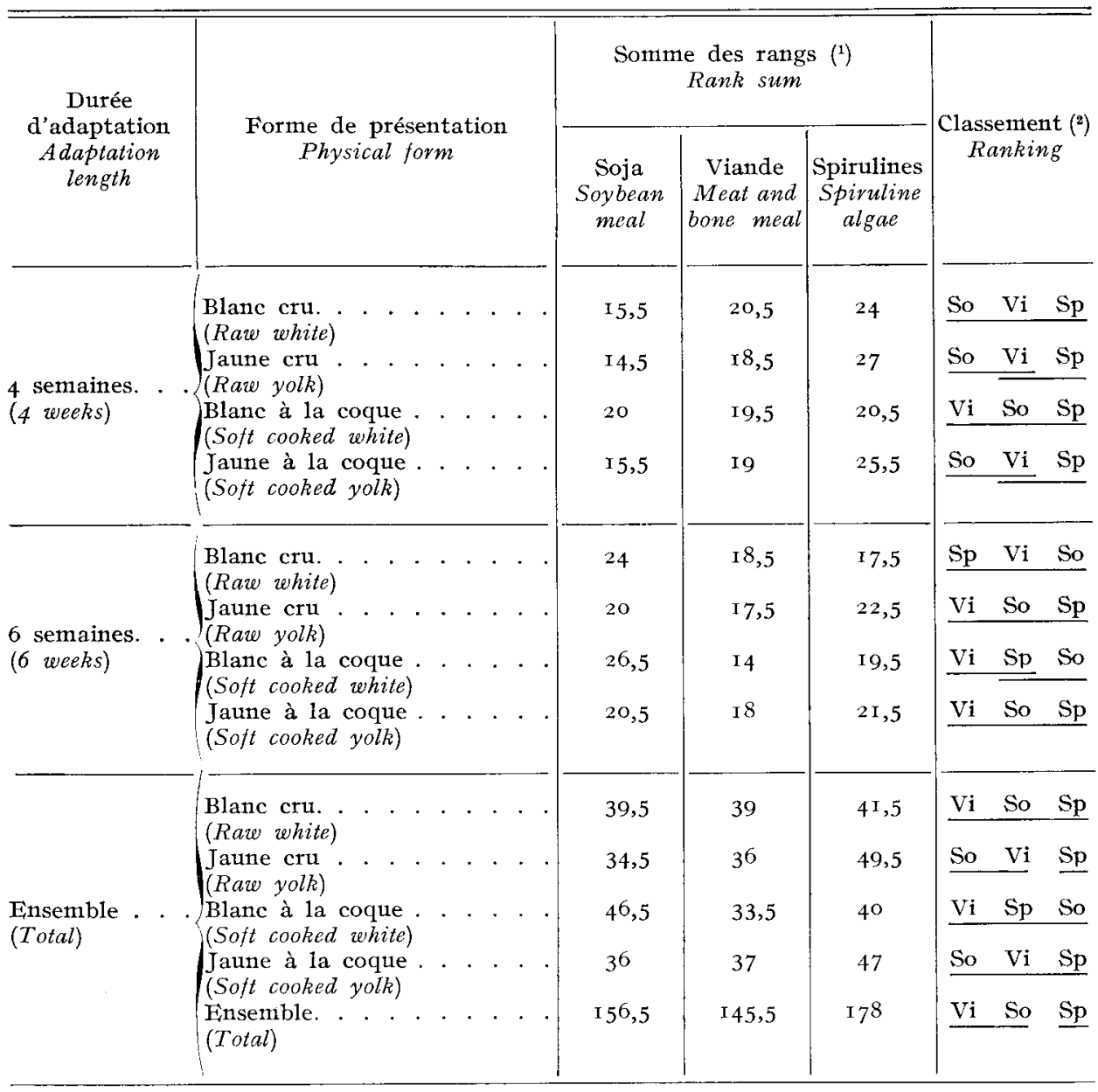

(1) et $\left({ }^{2}\right)$ Voir tableau $x($ See table $I)$; So $=$ Soja $($ Soybean meal $) ; \mathrm{Vi}=$ Viande $($ Meat and bone meal); $\mathrm{Sp}=$ Spirulines (Spiruline algae).

Effet de l'incorporation des légumineuses pois et féverole ( ${ }^{\text {re }}$ série, tabl. I et 2)

Les œufs pondus par les animaux témoins sont jugés moins francs que ceux pondus par les animaux recevant les 2 régimes expérimentaux (pois 30 p. roo et féverole I5 p. Ioo) :

- pour le blanc cru, le jaune à la coque et pour la somme des formes de présentation dans le cas du régime pois; 


\section{TABLEAU 6}

$3^{\mathrm{e}}$ série: caractéristiques sensorielles des aufs (1)

$3^{\text {rd }}$ experiment: sensory characteristics of eggs

\begin{tabular}{|c|c|c|c|c|c|}
\hline \multirow{2}{*}{$\begin{array}{c}\text { Forme } \\
\text { de présentation } \\
\text { Physical form }\end{array}$} & \multirow{2}{*}{$\begin{array}{c}\text { Régime } \\
\text { Diet }\end{array}$} & \multirow{2}{*}{$\begin{array}{l}\text { Nombre } \\
\text { d'cufs ne } \\
\text { présentant } \\
\text { aucun } \\
\text { défaut } \\
\text { Number } \\
\text { of eggs } \\
\text { without } \\
\text { taint }\end{array}$} & \multicolumn{2}{|c|}{$\begin{array}{l}\text { Nombre de défauts } \\
\text { et défauts principaux } \\
\text { Number of taints } \\
\text { and main taints }\end{array}$} & \multirow{2}{*}{$\begin{array}{l}\text { Appré- } \\
\text { ciation } \\
\text { globale }\left({ }^{3}\right) \\
\text { Palatability } \\
\text { score }\end{array}$} \\
\hline & & & $\begin{array}{c}\text { Odeur }\left({ }^{2}\right) \\
\text { Odour }\end{array}$ & $\begin{array}{c}\text { Flaveur }{ }^{(3)} \\
\text { Flavour }\end{array}$ & \\
\hline $\begin{array}{l}\text { Blanc cru. } \\
\text { (Raw white) }\end{array}$ & $\begin{array}{l}\text { Soja. } \\
\text { (Soybean meal) } \\
\text { Viande. } \\
\text { (Meat and bone meal) } \\
\text { Spirulines } \cdot \cdot \\
\text { (Spirmline algae) }\end{array}$ & $\begin{array}{l}7 \\
8 \\
8\end{array}$ & $\begin{array}{l}6 / 4 \text { poi } 2,5 \\
5 /- \\
4 /-\end{array}$ & $\begin{array}{l}20 / 6 \text { poi } 3,3 \\
\text { et } 5 \text { chi } 1,6 \\
\text { I } 4 /- \\
\text { I } 3 / 6 \text { poi } 3\end{array} \mid$ & $\begin{array}{l}3,4 \\
3,9 \\
3,7\end{array}$ \\
\hline $\begin{array}{l}\text { Jaune cru. } \\
(\text { Raw yolk })\end{array}$ & $\begin{array}{l}\text { Soja. } \\
\text { (Soybean meal) } \\
\text { Viande. } \\
(\text { Meat and bone meal) } \\
\text { Spirulines } \cdot \cdot \\
\text { (Spiruline algae) }\end{array}$ & $\begin{array}{r}\mathrm{I} 3 \\
\mathrm{I} 3 \\
6\end{array}$ & $\begin{array}{l}2 /- \\
2 /- \\
3 /-\end{array}$ & $\mid \begin{array}{l}7 /- \\
10 /- \\
20 /-\end{array}$ & $\begin{array}{l}4,9 \\
4,8 \\
3,6\end{array}$ \\
\hline $\begin{array}{l}\text { Blanc à la coque } \\
\text { (Soft cooked white) }\end{array}$ & $\begin{array}{l}\text { Soja. } \cdot \\
(\text { Soybean meal }) \\
\text { Viande. } \cdot \\
(\text { Meat and bone meal }) \\
\text { Spirulines } \cdot \cdot \\
(\text { Sfiruline algae })\end{array}$ & $\begin{array}{l}7 \\
\text { II } \\
\text { I I }\end{array}$ & $\begin{array}{l}8 / 3 \text { chi } I, 7 \\
2 /- \\
4 /-\end{array}$ & $\begin{array}{l}\text { I5/- } \\
9 /- \\
\text { Io/3 poi } 3\end{array}$ & $\begin{array}{l}3,7 \\
4,4 \\
4,2\end{array}$ \\
\hline $\begin{array}{l}\text { Jaune à la coque } \\
\text { (Sott cooked yolk) }\end{array}$ & $\left\{\begin{array}{l}\text { Soja. } \\
(\text { Soybean meal }) \\
\text { Viande. } \\
(\text { Meat and bone meal }) \\
\text { Spirulines } \cdot \\
(\text { Spiruline algae })\end{array}\right.$ & $\begin{array}{l}\text { I I } \\
\text { II } \\
6\end{array}$ & $\begin{array}{l}4 /- \\
2 /- \\
9 / 3 \text { chi I,7 }\end{array}$ & \begin{tabular}{|} 
Io $/ 3$ poi 2,3 \\
et 3 chi 2,3 \\
$9 /-$ \\
$22 / 8$ chi 2,8
\end{tabular} \mid & $\begin{array}{l}4,6 \\
4,8 \\
3,9\end{array}$ \\
\hline
\end{tabular}

(1) Valeurs obtenues à partir de 20 réponses (Data calculated from zo judgments).

$\left({ }^{2}\right)$ et $\left({ }^{3}\right)$ Voir tableau 2 (See table 2 ).

- pour le blanc cru uniquement dans le cas du régime féverole; aucune différence n'est observée entre les 2 régimes expérimentaux.

Pour le régime soja, le blanc d'œuf cru présente une odeur et une flaveur de poisson et le blanc d'œuf à la coque une flaveur de terre (tab1. 2). 


\section{TABLEAU 7}

Récapitulation des diffévences observées dans le classement des outs entre 4 et 6 semaines d'adaptation des animaux aux régimes

Differences observed in egg ranking between 4 and 6 weeks of adaptation of the hens to diets

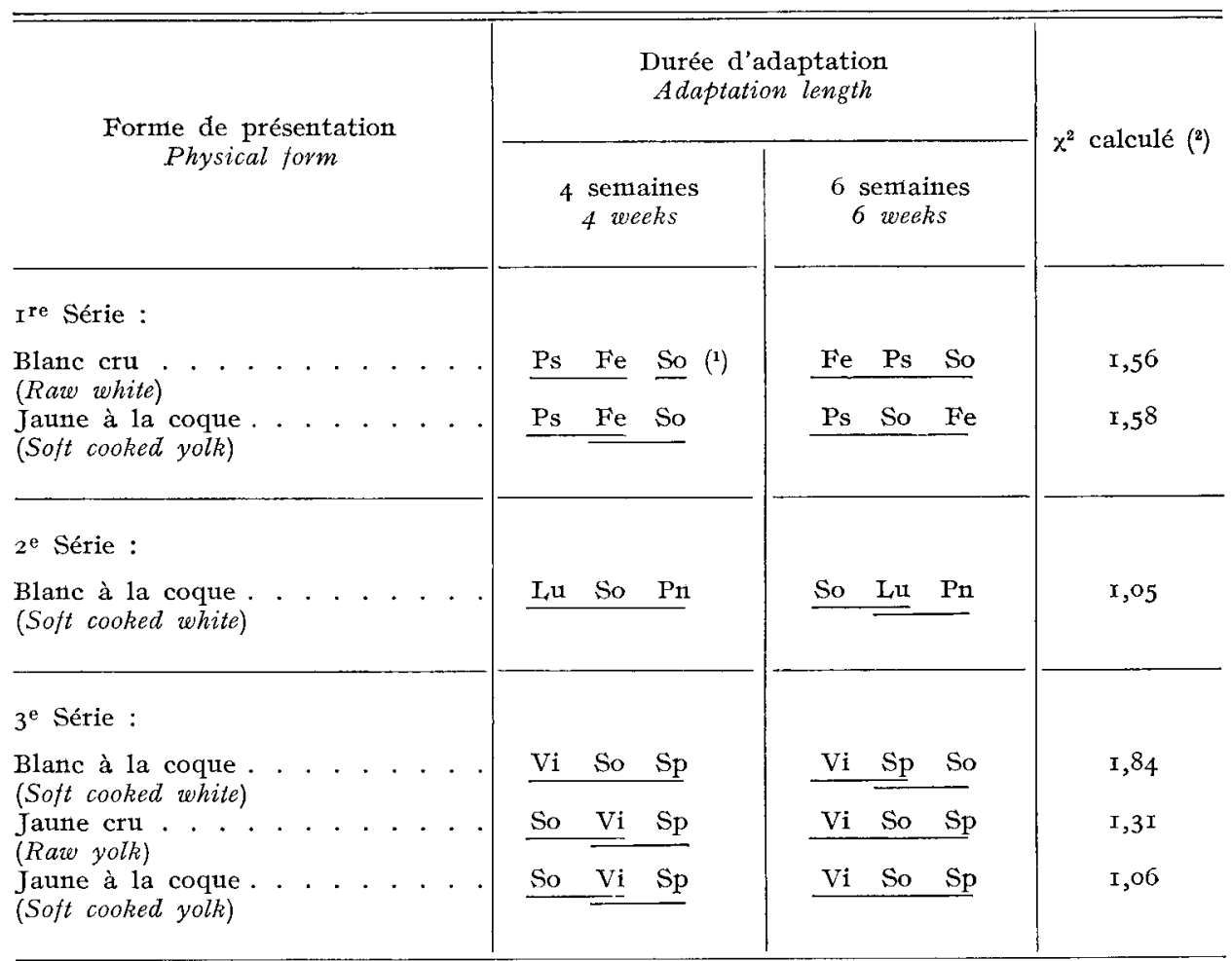

( $\left.{ }^{1}\right)$ Les essais soulignés d'un même trait ne diffèrent pas significativement $(P \leqslant 0,05)$ (Samples underscored by the same line do not differ significantly $(P \leqslant 0.05)$ ).

(2) Valeur du $\chi^{2}$ au seuil de $5 \mathrm{p}$. Ioo $=5,99$ (Value of $\chi^{2}$ at the $5 \mathrm{p}$. Ioo level $=5.99$ ).

Effet de l'incorporation de farine de poisson et de protéine de luzerne $\left(2^{\mathrm{e}}\right.$ série, tabl. 3 et 4 )

Pour les 4 formes de présentation, le même classement, Soja-Luzerne-Poisson, est observé; il est significatif seulement pour l'œuf à la coque (blanc et jaune). Pour la somme des formes de présentation, les 3 échantillons sont jugés différents, les oufs du régime témoin étant jugés les plus franes et ceux du régime poisson les moins francs.

Le groupe d'évaluation attribue aux cufs à la coque régime poisson (blanc et jaune) une note d'appréciation globale inférieure à celle des 2 autres échantillons (tab1. 4) Une odeur et une flaveur de poisson (intensité moyenne 2,2 à $2,8)$ sont détectées. 


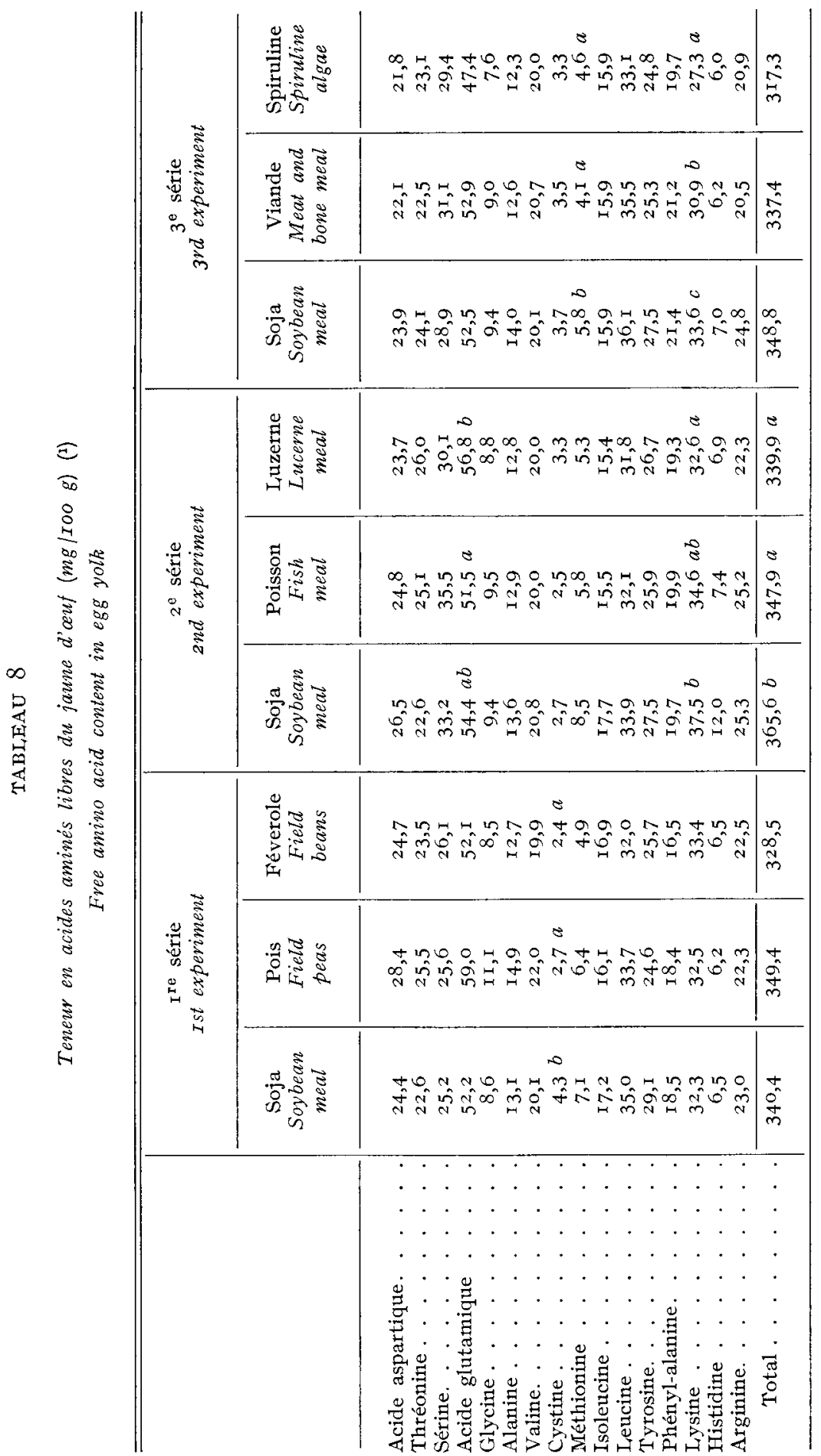


Effet de l'incorporation de farine de viande et d'algues Spirulines ( $3^{\mathrm{e}}$ série, tabl. 5 et 6 )

Les œufs pondus par les animatux recevant le régine Spirulines sont jugés moins francs que ceux pondus par les animaux recevant les 2 autres régimes (témoin et farine de viande), pour le jaune cru et pour la somme des formes de présentation. Les œufs pondus par les animaux recevant le régime farine de viande ne diffèrent pas des témoins.

Pour le jaune (cru et la coque) ces écarts en classement apparaissent au niveau des caractéristiques sensorielles. Le jaune d'œuf à la coque régime Spirulines présente une flaveur chimique caractéristique (intensité moyenne 2,8 ).

\section{Teneur du iaune en acides aminés libres}

Les résultats de chacune des 3 séries expérimentales sont rapportés dans le tabl. 8.

La teneur des jaunes en acides aminés libres totaux varie faiblement. Des variations sont observées entre régimes dans une même série, mais aussi entre les 3 régimes soja témoins. Les valeurs observées oscillent entre 3 I 7 et $366 \mathrm{mg} /$ Ioo g. Les teneurs les plus élevées sont en général observées pour les œufs des régimes témoins. Une différence significative avec les lots expérimentaux est observée seulement dans la deuxième série (farine de poisson et protéine de luzerne).

Les acides aminés libres les plus variables sont l'acide glutamique, la lysine et les acides aminés soufrés (méthionine et cystine). Dans chacune des 3 séries, la teneur en méthionine + cystine libres est plus élevée pour les œufs des régimes soja témoins que pour ceux des 2 régimes expérimentaux. Dans la deuxième série, les oufs du régime protéine de luzerne ont la plus forte teneur en acide glutamique et la plus faible en 1ysine. Dans la troisième série, les teneurs en lysine des cufs des 3 régimes sont différentes.

\section{Discussion}

Il est en général admis que la méthode du profil permet d'évaluer les échantillons indépendamment les uns des autres, en particulier de leur attribuer une note d'appréciation globale "absolue". Dans le cas présent, cette hypothèse n'est pas vérifiée (tabl. 9) : la note attribuée aux œufs pondus par les animaux témoins varie au cours des 3 séries expérimentales. Dans chaque série, elle est fonction des notes attribuées aux oufs des 2 lots expérimentaux : faible dans la première série, en présence de lots expérimentaux ayant des caractéristiques sensorielles franches, elle est élevée dans la deuxième série, en présence de lots expérimentaux présentant des défauts d'odeur ou de flaveur (effet dit de halo ou encore de contraste).

Les caractéristiques sensorielles des œufs du régime soja témoin restent cependant homogènes pour l'ensemble de l'expérimentation. En particulier, dans les trois séries, une odeur et une flaveur de poisson est toujours détectée dans le blanc cru. 
TABLEAU 9

Notes d'appréciation globale des auts pour l'ensemble des formes de présentation ( $\left.{ }^{1}\right)$ Palatability scoves of eggs for all physical forms studied

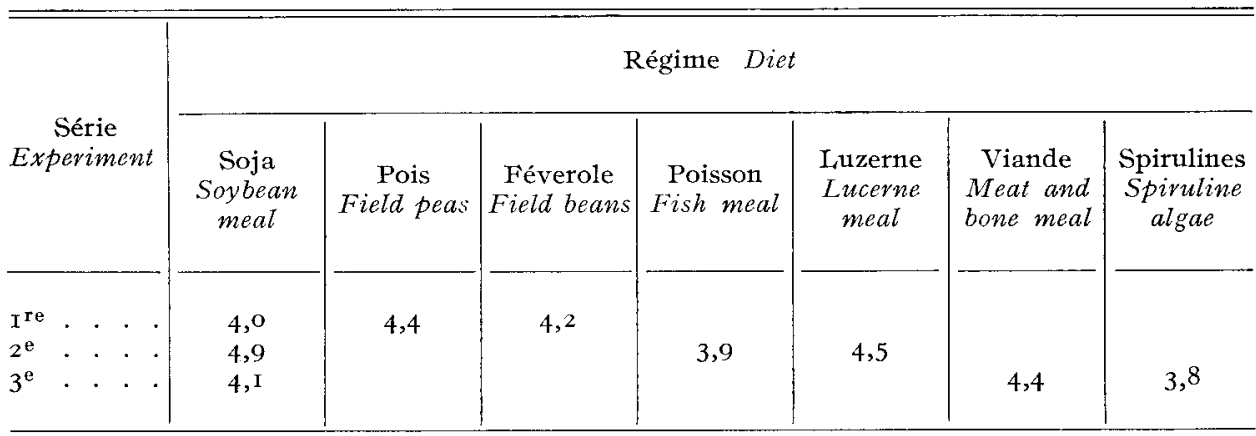

( $\left.{ }^{1}\right)$ Chaque valeur est la moyenne de 40 réponses ( ${ }^{\text {re }}$ série) et de 80 réponses $\left(2^{\mathrm{e}}\right.$ et $3^{\mathrm{e}}$ séries) (Each value is the average of 40 judgments (Ist experiment) and of 80 judgments (2nd and 3 rd experiments)).

En ce qui concerne l'influence de la forme de présentation sur les caractéristiques sensorielles des œufs, les résultats obtenus montrent que :

- la forme de présentation n'intervient pas pour le blanc : les différences entre échantillons sont observées aussi bien sur l'œuf cru que sur l'œuf à la coque. Cependant, le nombre de défauts caractéristiques décelés (odeur et flaveur) est plus élevé dans le blanc cru;

- la forme de présentation intervient pour le jaune : seule la forme à la coque permet d'observer des différences entre échantillons;

- pour tous les échantillons, la note d'appréciation globale du blanc cru est inférieure à celle des 3 autres formes; ce résultat peut s'expliquer, en partie tout au moins, par l'aversion éprouvée par les sujets pour cette forme de présentation.

Pour les niveaux d'incorporation pratiqués, les aliments protéiques utilisés modifient les caractéristiques sensorielles des œufs : des variations significatives sont observées, même si elles restent faibles. Ainsi l'incorporation dans la ration des animaux de Io p. Ioo de farine de poisson, de 6 p. Ioo de protéine de luzerne ou de II, 5 p. Ioo d'algues Spirulines entraîne la production d'œufs présentant des caractéristiques sensorielles différentes de celles des œufs produits par les animaux recevant le régime témoin, et moins appréciés. Inversement, l'incorporation de $30 \mathrm{p}$. roo de pois améliore les qualités sensorielles des œufs. Une tendance identique (mais non significative) est observée après incorporation dans la ration de I $_{5} \mathrm{p}$. Ioo de féverole ou de ro $\mathrm{p}$. Ioo de farine de viande.

En ce qui concerne l'utilisation des farines de poisson, ces résultats sont en accord avec les travaux de KOEHLER et BEARSE (I975) : ces auteurs observent que la flaveur des cufs est altérée lorsque le taux d'incorporation de farine de poisson atteint 5 p. roo de la ration des poules pondeuses. Pour les autres sources protéiques étudiées, il n'existe pas de travaux antérieurs sur l'influence de leur incorporation sur les caractéristiques sensorielles des œufs produits. 
Au niveau des acides aminés libres, les variations sont faibles, tant aux niveaux qualitatif que quantitatif, et peuvent difficilement expliquer 1'apparition de flaveurs anormales dans les jaunes. Ainsi, une odeur ou une flaveur sulfureuse ne peut être associée avec une augmentation de la teneur en méthionine + cystine.

Accepté pour publication en juin 1979.

\title{
Remerciements
}

Les travaux rapportés ont été réalisés avec l'aide financière de la D.G.R.S.T., Action Concertée Technologie Alimentaire et Agricole, décisions no 77-7-0605 et 77-7-06o6. Les Sociétés France-Luzerne et Sosa Texcoco doivent être remerciées pour la fourniture respective des protéines de luzerne $\mathrm{P} \times \mathrm{I}$ et des algues Spirulines.

\section{Summary}

\author{
Dietary proteins and egg quality \\ II. - Influence of the protein source added to laying hen diets \\ on egg flavour and tree amino acid content of egg yolk
}

Experiments were monitored to study the influence of a partial substitution of some new protein sources for soybean meal in Warren hen rations on the odour and flavour of eggs and on the free amino acid content of egg yolk.

Six experimental diets were divided into 3 series:

- field peas ( 30 p. IOo) and field beans ( 15 p. roo);

- fish meal (Io p. roo) and lucerne meal (6 p. roo);

- meat and bone meal (Io p. Ioo) and spiruline algae (I I.5 p. Ioo).

Every serie includes a control soybean diet. A trained panel evaluated raw and softcooked egg whites and yolks using an organoleptic descriptive analysis (fig. I).

Results obtained indicate that:

I. The way of preparing eggs influenced their sensory characteristics: in the white, differences between the diets were observed both in raw and soft-cooked eggs; in the yolk, differences were observed only in soft-cooked eggs.

2. Fish meal, lucerne meal and Spiruline algae significantly spoiled the sensory characteristics of eggs as compared with control eggs; whites and yolks from eggs layed by hens receiving the fish meal diet exhibited a fishy odour and flavour (table 4); Spiruline algae diet resulted in yolks with a " chemical" flavour (table 6). On the contrary, field peas improved the sensory characteristics of the eggs (table 2). Field beans and meat and bone meal had no significant influence on the sensory characteristics of the eggs.

3. The experimental diets only slightly affected the free amino acid content of the yolks (table 8) and no relationship was established between these variations and an abnormal odour or flavour of the egg yolks.

\section{Références bibliographiques}

Hawrysh Z. J., Clandinin D. R., Robblefe A. R., Hardin R. T., Darlington K., I975. Influence of rapeseed meal on the odor and flavor of eggs from different breeds of chickens. J. Inst. Can. Sci. Technol. Aliment., 8, 51-54.

HIPPE R. L., WARTHESEN J. J., I978. Effect of methionine and N-acetylmethionine fortification on the flavor of soy bread and soy milk. J. Fd. Sci., 43, 793-796. 
KOEHLER H. H., BEARSE G. E., 1975. Egg flavor quality as affected by fish meals or fish oils in laying rations. Poultry Sci., 54, 88I-889.

KOEHLER H. H., JACOBSON M., 1972. Flavor changes in egg white and yolk resulting from various storage conditions. Washington Agricultural Experiment Station Bulletin, 760, I-6.

Kramer A., Kahan G, CoOper D., Papavasiliou A., i974. A non-parametric ranking method for the statistical evaluation of sensory data. Chemical Senses and Flavor, 1, I2I-I33.

LARBIER M., I973. Recherche sur la signification des acides aminés libres présents dans le jaune de l'cuf chez Gallus gallus. Thèse doctorat $3^{\mathrm{e}}$ cycle Université Paris-VI, $4^{6}$ pages.

IfEeson S., Summers J. D., 1978. Dietary gums and fishy odours in eggs. Poultry Sci., 57, 3I 4 -3 I 5 .

MOORE S., SPACKMAN D. H., STEIN W. H., I $95^{8}$. Chromatography of amino-acids on sulfonated polystyrene resins. Analyt. Chem., 30, I $185^{-1} 190$.

OVERFIELD N. D., ELSON H. A., I975. Dietary rapeseed meal and the incidence on tainted eggs. Br. Poult. Sci., 16, 2I3-2I 7 .

SaUveur B., Zybiko A., Colas B., I979. Protéines alimentaires et qualité de l'œuf. I. Effet de quelques protéines sur la qualité interne de l'œuf et les propriétés fonctionnelles. $A n n$. Zootech., 28, 27I-295.

Solms J., I969. The taste of amino-acids, peptides and proteins. J. Agr. Food Chem., 17, 686-688. 